\title{
NÍVEIS DE CÁLCIO E GRANULOMETRIAS DO CALCÁRIO PARA FRANGAS DE REPOSIÇÃO NO PERÍODO DE 3 A 16 SEMANAS DE IDADE ${ }^{1}$
}

\author{
Calcium levels and particle size of limestone for \\ replacement pullets the period of 3 to 16 weeks of age
}

\author{
Adriano Geraldo ${ }^{2}$, Antônio Gilberto Bertechini ${ }^{3}$, \\ Luís David Solis Murgas ${ }^{4}$, Elias Tadeu Fialho ${ }^{5}$, Joel Augusto Muniz ${ }^{6}$
}

\begin{abstract}
RESUMO
Realizou-se um experimento com o objetivo de avaliar os efeitos dos níveis nutricionais de cálcio e granulometrias do calcário em rações de frangas de reposição sobre o desenvolvimento corporal e morfométrico do trato digestório. Foram utilizadas 1400 frangas de reposição da linhagem Lohmann LSL, empregando-se DIC em esquema fatorial 5x2 (níveis de cálcio e granulometrias do calcário), com 5 repetições com 28, 18 e 16 aves por parcela, respectivamente, nos períodos de 3 a 7, 8 a 12 e 13 a 16 semanas de idade. As dietas experimentais foram à base de milho e farelo de soja suplementadas com minerais, vitaminas e fitase (500 FTU/kg), sendo isoprotéicas, isocalóricas e isofosfóricas, tendo como fonte de fósforo o fosfato monoamônio e balanceadas de acordo com o Manual da linhagem Lohmann LSL (LOHMANN, 1999). Utilizou-se o calcário em granulometrias fina $(\mathrm{DGM}=0,135 \mathrm{~mm})$ e grossa $(\mathrm{DGM}=0,899 \mathrm{~mm})$ para atender os níveis de 0,$60 ; 0,75 ; 0,90 ; 1,05 \mathrm{e} 1,20 \%$ de cálcio total na dieta. Avaliaram-se o desempenho no período de 3 a 16 semanas, morfometria do trato digestório e teores de cinzas e minerais na tíbia de aves de cada parcela abatida na $16^{\mathbf{a}}$ semana de idade. O consumo de ração apresentou aumento linear com a elevação dos níveis de cálcio. Não houve diferenças significativas do ganho de peso, conversão alimentar e porcentagem do trato digestório. Obtiveram-se maiores teores de cinzas e cálcio em frangas que receberam a granulometria grossa nos níveis de 0,90 e $1,05 \%$ de cálcio. Conclui-se que o nível de cálcio ideal para frangas nas fases de cria e recria é de $0,90 \%$ e a granulometria do calcário deve ser a grossa $(\mathrm{DGM}=0,899 \mathrm{~mm})$.
\end{abstract}

Termos para indexação: Ave de postura, nutrição de monogástricos, cálcio, morfometria, trato digestório.

\section{ABSTRACT}

The present work was performed with the purpose of evaluating the effects of the nutritional levels o calcium and particle sizes of limestone replacement pullet rations on the body and morphometric development of the digestive tract. 1,400 replacement pullets of the Lohmann LSL strain, employing CRD in a 5 x 2 factorial scheme (calcium levels and particle size of limestone) with five replicates with 28, 18 and 16 birds per plot, respectively over the periods of 3 to 7,8 to 12 and 13 to 16 weeks of age. The experimental diets were based on corn, soybean meal and wheat meal (wheat only at growing phase) supplemented with minerals, vitamins and phytase $(500 \mathrm{FTU} / \mathrm{Kg})$, their being isoprotein, isocaloric and isophosphorus, their having as a phosphorus source monoammonium phosphorus and balanced according to the Lohmann LSL strain Handbook (LOHMANN, 1999), supplemented with limestone in fine $(\mathrm{DGM}=0.135 \mathrm{~mm})$ and gross $(\mathrm{DGM}=0.899$ $\mathrm{mm}$ ) particle size to meet the levels of $0.60,0.75,0.90,1.05$ and $1.20 \%$ of total calcium in the diet. The performance in the period of 3 to 16 weeks , morphometry of the digestive tract and contents of ashes and minerals in the birds' tibia of each plot slaughtered on the 16th week of age. Ration consumption in the period presented a linear growth with the rise of calcium levels. There were no significant differences of weight gain, feed conversion and \% digestive tract. On the 16th week of age, higher ash and calcium contents were achieved in pullets were fed gross particle size at the levels of 0.90 and $1.05 \%$ of calcium. In the initial and growing phases the level of $0.90 \%$ Ca proved ideal in gross size particle for the osseous formation.

Index terms: Hens, poultry nutrition, calcium, morfometric, digestory tract.

(Recebido para publicação em 7 de abril de 2003 e aprovado em 23 de setembro de 2003)

\footnotetext{
1. Parte dos resultados de dissertação do primeiro autor, projeto financiado pelo CNPq.

2. Zootecnista, mestrando do Departamento de Zootecnia da Universidade Federal de Lavras/UFLA - Caixa Postal 37 - 37200-000 - Lavras, MG.

3. Zootecnista, Professor Titular do Departamento de Zootecnia/UFLA.

4. Médico Veterinário, Professor Adjunto do Departamento de Medicina Veterinária/UFLA.

5. Zootecnista, Professor Titular do Departamento de Zootecnia /UFLA.

6. Engenheiro Agrônomo, Professor Titular do Departamento de Ciências Exatas/UFLA.
} 


\section{INTRODUÇÃO}

O grande desenvolvimento genético das aves de postura nos últimos anos tem alterado aspectos fisiológicos importantes nessas aves, havendo a necessidade de novas pesquisas para equacioná-los.

Em razão da maior demanda na eficiência produtiva, existe hoje a necessidade de se produzir frangas de reposição que possa refletir, na sua fase de produção, todo seu potencial genético para utilizá-lo com grande eficiência.

Vários trabalhos têm sido realizados para avaliar a granulometria ideal do calcário a ser fornecido para poedeiras para obter melhor desempenho e qualidade dos ovos, e maiores partículas da fonte de cálcio têm proporcionado maior retenção na parte superior do trato digestório, disponibilizando o cálcio vagarosa e uniformemente durante o período de formação da casca do ovo (LEESON e SUMMERS, 1997) e aumentando a retenção nos ossos medulares das poedeiras (RAO e ROLAND, 1990). Em relação a frangas de reposição, não foram encontrados na literatura trabalhos nos quais se avaliaram os efeitos das granulometrias da fonte de cálcio sobre o desempenho.

O conhecimento dos níveis ideais de cálcio para cada fase do desenvolvimento da ave é de suma importância, visto que esse macromineral é essencial para a formação e manutenção do esqueleto e que seu excesso na dieta pode interferir na disponibilidade de outros minerais como o fósforo, magnésio, manganês e zinco, além de tornar a dieta menos palatável e diluir outros componentes presentes, quando altos níveis da fonte carbonato de cálcio (calcário) são utilizados.

O consumo de ração pelas aves pode ser afetado pelo cálcio que, possivelmente, parece agir no hipotálamo, sendo ainda não conhecido se sua ação é direta ou indireta, pois ele induz a liberação de norepinefrina, mediador que atua no sistema nervoso central, propiciando aumento no consumo de ração (BORGES, 1999).

Estudos com cálcio $(\mathrm{Ca})$ e fósforo $(\mathrm{P})$ têm sido baseados em ganho de peso, conversão alimentar, cinza e resistência óssea à quebra (BAILEY et al., 1986), e o tamanho esquelético obtido no desenvolvimento da franga pode determinar as reservas de $\mathrm{Ca}$ da ave no pico de produção de ovos (COELHO, 2001).

Pesquisas têm sido realizadas ao longo dos anos para estimar a exigência nutricional de cálcio para poedeiras, havendo ainda contradições quanto ao melhor nível de $\mathrm{Ca}$ a ser utilizado na dieta de frangas de reposição, uma vez que têm sido encontrados valores variáveis entre
0,6 a 1,2\% de cálcio (BERG et al., 1964; ANDERSON, 1967; GERRY e BIRD, 1967; MEYER et al., 1971; ALBINO et al., 2001).

Assim, verifica-se a necessidade de se determinar os níveis nutricionais de cálcio e granulometrias do calcário para frangas nas fases de cria e recria, seus efeitos sobre o desenvolvimento corporal e morfométrico do trato digestório.

\section{MATERIAL E MÉTODOS}

O experimento foi conduzido no Setor de Avicultura do Departamento de Zootecnia da Universidade Federal de Lavras. Foram utilizadas 1400 aves da linhagem comercial Lohmann-LSL com 1 dia de idade, criadas em círculos de proteção até 14 dias e submetidas a uma mesma ração com $0,94 \%$ de Ca com granulometria do calcário com diâmetro geométrico médio (DGM) igual a $0,135 \mathrm{~mm}$, sendo, então, pesadas e transferidas para baterias $(94 \times 94 \mathrm{~cm}$ ), iniciando o fornecimento dos tratamentos a partir dessa data. Foi estudada uma fonte de calcário em duas granulometrias (DGM $=0,135 \mathrm{~mm}$ e DGM $=0,899 \mathrm{~mm})$, fornecida em cinco níveis $(0,60 \% ; 0,75 \% ; 0,90 \% ; 1,05 \%$ e $1,20 \%$ de cálcio), constituindo-se num fatorial 2 x 5 com dez tratamentos instalados num delineamento inteiramente casualizado com cinco repetições. As parcelas experimentais constituíram-se de 28 aves por bateria no período de 3 a 7 semanas de idade; 18 aves (2 gaiolas de recria contendo 9 aves cada uma) no período de 8 a 12 semanas de idade e 16 aves ( 2 gaiolas de recria com 8 aves cada uma) no período de 13 a 16 semanas de idade, quando as aves foram criadas em galpão de recria.

As práticas de manejo normais incluíram debicagens e vacinações de acordo com os principais desafios da região.

As dietas experimentais foram formuladas à base de milho e farelo de soja, suplementadas com minerais, vitaminas e fitase (500 FTU/kg ração cuja função é disponibilizar para as aves o fósforo complexado nas moléculas de fitato presente nos grãos), sendo isoprotéicas, isocalóricas e isofosfóricas, de acordo com as recomendações do Manual de Criação e Manejo da linhagem Lohmann LSL (LOHMANN, 1999), sendo a composição das matérias-primas as tabeladas em Rostagno et al. (2000). A fonte de fósforo utilizada na formulação das rações experimentais no período de 3 a 16 semanas de idade foi o fosfato monoamônio purificado (MAP) contendo 27,0\% de fósforo disponível. Foi utilizado um programa de alimentação com ra-

Ciênc. agrotec., Lavras, v. 28, n. 2, p. 437-442, mar./abr., 2004 
ção de 1 a 2 semanas, cria no período de 3 a 7 semanas e recria de 8 a 16 semanas, suplementadas com colina, segundo recomendações de Rostagno et al. (2000).

As características avaliadas nos períodos de 3 a 16 semanas de idade foram ganho de peso (g/ave no período), conversão alimentar (g ração/g PV) e consumo (g/ave no período). Avaliaram-se nas aves abatidas na $16^{\mathrm{a}}$ semana de idade, segundo a metodologia descrita por Choi et al. (1986), a porcentagem do trato digestório (do proventrículo até o reto, incluindo os cecos e pâncreas) em relação ao peso vivo (PV) da ave e o comprimento do intestino delgado ( $\mathrm{cm} / \mathrm{kg} \mathrm{PV})$, sendo as aves abatidas submetidas a um jejum de 12 horas no período noturno anterior ao abate. Foi realizada a medição do comprimento do metatarso (cm/kg PV), determinação dos teores de cinzas (\%), cálcio (\%) e fósforo (\%) na tíbia das aves abatidas, sendo os resultados expressos na base de matéria seca desengordurada, segundo AOAC (1990).

Os resultados obtidos foram submetidos à análise estatística com a utilização do software Sistema de análises de variância para dados balanceados (SISVAR) descrito por Ferreira (2000).

\section{RESULTADOS E DISCUSSÃO}

Os valores médios de ganho de peso, consumo de ração e conversão alimentar no período de 3 a 16 semanas de idade são apresentados na Tabela 1 .

Não houve interação significativa $(\mathrm{P}>0,05)$ e nem efeito independente $(\mathrm{P}>0,05)$ dos níveis de cálcio e das granulometrias do calcário sobre o ganho de peso. Os resultados estão de acordo com Berg et al. (1964), que também não encontraram diferenças no ganho de peso na $12^{\underline{a}}, 16^{\underline{a}}$ e $21^{\underline{a}}$ semana de idade de frangas alimentadas com dieta contendo 0,$66 ; 1,12$ e 2,01\% de cálcio no período de 8 a 21 semanas. Simco e Stephenson (1961) também não encontraram diferenças no ganho de peso de frangos de corte alimentados com dietas contendo 0,$50 ; 0,60 ; 0,80$ e $1,0 \%$ de cálcio no período de 1 a 8 semanas de idade, e apenas o nível de $0,15 \%$ de cálcio afetou o ganho de peso. Meyer et al. (1971) também não observaram diferenças no peso corporal no período de 8 a 20 semanas de idade de aves de linhagem para postura machos e fêmeas que receberam dieta com 0,$40 ; 0,70 ; 0,90 ; 1,20$ e 1,5\% de cálcio. Gerry e Bird (1967) também não encontraram diferenças no ganho de peso de frangas que receberam 0,$80 ; 1,16 ; 2,4 \mathrm{e}$ $3,2 \%$ de cálcio no período de 10 a 24 semanas de idade.

TABELA 1 - Médias de ganho de peso (g), consumo de ração (g) e conversão alimentar no período de 3 a 16 semanas de idade, segundo os níveis de cálcio e granulometrias do calcário.

\begin{tabular}{ccccccc}
\hline \multirow{2}{*}{ Nível Ca } & \multicolumn{2}{c}{ Ganho de peso } & \multicolumn{2}{c}{ Consumo $^{\mathbf{1}}$} & \multicolumn{3}{c}{ CA } \\
\cline { 2 - 7 } & $\mathbf{0 , 1 3 5} \mathbf{~ m m}$ & $\mathbf{0 , 8 9 9} \mathbf{~ m m}$ & $\mathbf{0 , 1 3 5} \mathbf{~ m m}$ & $\mathbf{0 , 8 9 9} \mathbf{~ m m}$ & $\mathbf{0 , 1 3 5} \mathbf{~ m m}$ & $\mathbf{0 , 8 9 9} \mathbf{~ m m}$ \\
\hline 0,60 & 980 & 962 & 4535 & 4510 & 4,65 & 4,71 \\
0,75 & 936 & 938 & 4600 & 4558 & 4,99 & 4,87 \\
0,90 & 1011 & 1099 & 4589 & 4562 & 4,56 & 4,23 \\
1,05 & 977 & 1068 & 4698 & 4574 & 4,82 & 4,30 \\
1,20 & 991 & 998 & 4619 & 4593 & 4,71 & 4,65 \\
\hline Média $^{2}$ & 979 & 1013 & 4608 a & $4559 \mathrm{~b}$ & 4,74 & 4,55 \\
\hline CV(\%) & \multicolumn{7}{c}{9,90} & \multicolumn{2}{c}{1,60} \\
\hline
\end{tabular}

${ }^{1}$ Efeito linear $(P=0,064) ; Y=148,91 x+4449,86\left(R^{2}=70,76 \%\right)$.

${ }^{2}$ Médias seguidas por letras diferentes na linha diferem pelo Teste $\mathbf{F}(\mathbf{P}<0,05)$. 
Houve somente o efeito dos níveis de cálcio da ração $(\mathrm{P}=0,064)$ sobre o consumo das frangas, proporcionando aumento linear do consumo com a elevação dos níveis de cálcio.

Albino et al. (2001) não encontraram diferenças no consumo de frangas leves e semipesadas alimentadas com 5 níveis de cálcio, variando de 0,60 a 1,00\% de cálcio no período de 7 a 12 semanas de idade. Berg et al. (1964) também não encontraram diferenças dos níveis de 0,$66 ; 1,12$ e 2,01\% de cálcio na ração sobre o consumo de frangas no período de 8 a 21 semanas de idade. Schoulten (2001) não encontrou diferenças no consumo de frangos de corte de 1 a 21 dias que receberam níveis de $0,43 \%$ a $1,30 \%$ de cálcio. $\mathrm{O}$ aumento no consumo em função do aumento nos níveis de cálcio pode ser devido ao estímulo no consumo propiciado pelo cálcio, o qual, segundo Borges (1999), possivelmente tenha ação no hipotálamo (ainda é desconhecido se a ação do cálcio é direta ou indireta), induzindo à liberação de norepinefrina, mediador que atua no sistema nervoso central, aumentando a ingestão de alimentos.

Não ocorreram interação significativa $(\mathrm{P}>0,05)$ e nem efeitos independentes $(\mathrm{P}>0,05)$ dos níveis de cálcio e granulometrias do calcário para a conversão alimentar.

Os resultados morfométricos (\% trato digestório, comprimento do intestino delgado e comprimento do metatarso) de frangas com 16 semanas de idade estão apresentados na Tabela 2.

Não foram constatados interação significativa $(\mathrm{P}>0,05)$ e efeito independente $(\mathrm{P}>0,05)$ dos níveis de cálcio e granulometrias do calcário sobre o desenvolvimento do trato digestório na $16^{\mathrm{a}}$ semana de idade.

Houve interação significativa $(\mathrm{P}<0,05)$ entre os níveis de cálcio e as granulometrias do calcário. Estudando a granulometria do calcário dentro de cada nível de cálcio, nota-se que as médias diferiram apenas no nível de $0,90 \%$ de cálcio, e a granulometria de DGM = $0,135 \mathrm{~mm}$ proporcionou maior comprimento do intestino delgado/kg de peso vivo.

Não houve interação significativa $(\mathrm{P}>0,05)$ e efeitos independentes $(\mathrm{P}>0,05)$ dos níveis de cálcio e das granulometrias do calcário sobre o comprimento do metatarso (canela), indicando serem os níveis e granulometrias utilizados suficientes para bom desenvolvimento corporal da ave. Os resultados estão de acordo com Meyer et al. (1971), que também não encontraram efeito dos níveis de 1,2 e $0,4 \%$ de cálcio fornecido no período de 12 semanas de idade, ao início de postura, sobre o comprimento do metatarso para aves macho e fêmea da linhagem de postura.

Os resultados do teor de cinzas, cálcio e fósforo na tíbia das aves na $16^{\mathrm{a}}$ semana de idade estão apresentados na Tabela 3.

TABELA 2 - Porcentagem do trato digestório (TD), comprimento do intestino delgado (CID e comprimento do metatarso (CM) de frangas de reposição com 16 semanas de idade.

\begin{tabular}{|c|c|c|c|c|c|c|}
\hline \multirow{2}{*}{ Nível Ca } & \multicolumn{2}{|c|}{$\operatorname{TD}(\%)$} & \multicolumn{2}{|c|}{ CID $(\mathrm{cm} / \mathrm{kg})$} & \multicolumn{2}{|c|}{$\mathrm{CM}(\mathrm{cm} / \mathbf{k g})$} \\
\hline & $0,135 \mathrm{~mm}$ & 0,899 mm & $0,135 \mathrm{~mm}$ & $0,899 \mathrm{~mm}$ & $0,135 \mathrm{~mm}$ & $0,899 \mathrm{~mm}$ \\
\hline 0,60 & 6,76 & 6,69 & 100 & 100 & 9 & 9 \\
\hline 0,75 & 6,57 & 6,66 & 100 & 107 & 8 & 9 \\
\hline $0,90^{1}$ & 7,00 & 6,43 & $106 \mathrm{a}$ & $96 \mathrm{~b}$ & 8 & 8 \\
\hline 1,05 & 6,89 & 6,77 & 106 & 98 & 9 & 8 \\
\hline 1,20 & 6,57 & 6,44 & 100 & 98 & 8 & 8 \\
\hline Média & 6,76 & 6,60 & 102 & 100 & 8 & 8 \\
\hline $\mathrm{CV}(\%)$ & \multicolumn{2}{|c|}{6,89} & \multicolumn{2}{|c|}{7,18} & \multicolumn{2}{|c|}{8,14} \\
\hline
\end{tabular}

\footnotetext{
${ }^{1}$ Médias seguidas por letras diferentes na linha diferem pelo Teste $\mathbf{F}(\mathbf{P}<0,05)$.
}

Ciênc. agrotec., Lavras, v. 28, n. 2, p. 437-442, mar./abr., 2004 
Houve interação significativa $(\mathrm{P}<0,05)$ entre os níveis de cálcio e as granulometrias do calcário sobre os teores de cinzas e cálcio da tíbia, não ocorrendo efeito dos níveis de cálcio dentro das granulometrias fina e grossa $(\mathrm{P}>0,05)$. Foi observado, ao se fazer o estudo de granulometria do calcário em cada nível de cálcio, que as médias diferiram apenas no nível de 0,90 e $1,05 \%$ de cálcio, e partículas com DGM $=0,899 \mathrm{~mm}$ proporcionaram maior acúmulo de cinzas na tíbia. Pode-se observar que nesse período ocorre o desenvolvimento dos órgãos reprodutores e, conseqüentemente, a secreção de estrógeno, que atua no aproveitamento do cálcio e no desenvolvimento dos ossos medulares que irão dar suporte para a calcificação da casca do ovo no período de produção de ovos. Os níveis de 0,90 e $1,05 \%$ de cálcio e o calcário de granulometria mais grossa foram favoráveis a um maior acúmulo de cinzas e cálcio nos ossos nesse período, sendo essa granulometria solubilizada mais lentamente e o cálcio disponibilizado durante o período noturno, em que as aves estão sem alimentação e a exigência de cálcio é mais elevada por causa da formação dos ossos medulares.

Berg et al. (1964) encontraram menor teor de cinzas na tíbia de frangas com 21 semanas de idade quando alimentadas com $2,01 \%$ de cálcio a partir da $8^{\mathrm{a}}$ semana de idade, se comparados com os níveis de 0,66 e $1,12 \%$ de cálcio.

Gerry e Bird (1967) observaram que as cinzas nos ossos tiveram pequeno decréscimo com o aumento do cálcio na dieta de crescimento, sendo os níveis utilizados no período de 10 a 24 semanas de idade de 0,$80 ; 1,60 ; 2,40$ e $3,20 \%$ de cálcio.

$\mathrm{O}$ teor de fósforo na tíbia não se alterou em nenhum tratamento, não ocorrendo interação entre níveis de cálcio e granulometrias do calcário $(P>0,05)$ e nem efeitos independentes $(P>0,05)$.

TABELA 3 - Teores de cinzas (\%), de cálcio (\%) e de fósforo (\%) na tíbia seca e desengordurada de aves com 16 semanas de idade, segundo os níveis de cálcio e as granulometrias do calcário.

\begin{tabular}{ccccccc}
\hline \multirow{2}{*}{ Nível Ca } & \multicolumn{2}{c}{ Cinzas } & \multicolumn{2}{c}{ Ca } & \multicolumn{2}{c}{ P } \\
\cline { 2 - 7 } & $\mathbf{0 , 1 3 5 m m}$ & $\mathbf{0 , 8 9 9} \mathbf{m m}$ & $\mathbf{0 , 1 3 5 m m}$ & $\mathbf{0 , 8 9 9 m m}$ & $\mathbf{0 , 1 3 5 m m}$ & $\mathbf{0 , 8 9 9 m m}$ \\
\hline 0,60 & 58,56 & 58,61 & 21,90 & 21,90 & 10,91 & 10,85 \\
0,75 & 58,51 & 58,28 & 22,11 & 21,75 & 10,92 & 10,71 \\
0,90 & $* 57,57 \mathrm{~b}$ & $59,24 \mathrm{a}$ & $* * 21,51 \mathrm{~b}$ & $22,34 \mathrm{a}$ & 10,61 & 11,03 \\
1,05 & $* * * 5,57 \mathrm{~b}$ & $60,19 \mathrm{a}$ & $* 21,59 \mathrm{~b}$ & $22,46 \mathrm{a}$ & 10,81 & 11,01 \\
1,20 & 58,40 & 58,73 & 21,71 & 22,06 & 10,97 & 10,85 \\
\hline Média & 58,12 & 59,07 & 21,76 & 22,10 & 10,84 & 10,89 \\
\hline CV(\%) & \multicolumn{7}{c}{2,36} & & & & 3,10 & \\
\hline
\end{tabular}

Médias seguidas por letras diferentes na linha diferem pelo Teste $\mathrm{F}(\mathrm{P}<0,05)^{*} \mathrm{e}(\mathrm{P}<0,01)^{* *}$. 


\section{CONCLUSÃO}

O nível ideal de cálcio para frangas de reposição no período de 3 a 16 semanas de idade é de $0,90 \%$ e a granulometria do calcário deve ser grossa $(\mathrm{DGM}=$ $0,899 \mathrm{~mm})$.

\section{REFERÊNCIAS BIBLIOGRÁFICAS}

ALBINO, L. F.; ROSTAGNO, H. S.; CARVALHO, D. C. O.; CUPERTINO, E. S.; TOLEDO, R. S. Níveis nutricionais de cálcio para frangas de reposição leves e semipesadas de 7 a 12 semanas de idade. In: REUNIÃO DA SOCIEDADE BRASILEIRA DE ZOOTECNIA, 2001, Piracicaba. Anais... Piracicaba: [s.n.], 2001. v. 38, p. 821-822.

ANDERSON, D. L. Pre-laying nutritional and environmental factors in the performance of the adult fowl. Poultry Science, Champaign, v. 46, n. 1, p. 5263, Jan. 1967.

ASSOCIATION OF OFFICIAL AGRICULTURAL CHEMISTS. Official methods of analysis of the Association of Official Analytical Chemists. 15. ed. Arlington, 1990. v. 1.

BAILEY, C. A.; LINTON, S.; BRISTER, R.; CREGER, C. R. Effects of gradel of dietary phosphorus on bone mineralization in the very young poult. Poultry Science, Champaign, v. 65, n. 5, p. 1018-1020, May 1986.

BERG, L. R.; BEARSE, G. E.; MERRILL, L. H. The calcium and phosphorus requirements of White Leghorn pullets from 8-21 weeks. Poultry Science, Champaign, v. 43, n. 4, p. 885-896, Apr. 1964.

BORGES, A. L. C. C. Controle da ingestão de alimentos. Cadernos Técnicos da Escola de Veterinária da UFMG, Belo Horizonte, v. 27, p. 67-79, 1999.

CHOI, J. H.; SO, B. S.; RYU, K. S.; KANG, L. Effects of pelleted or crumbled diets on the performance and the development of the digestive organs of broilers. Poultry Science, Champaign, v. 65, n. 3, p. 594-597, Mar. 1986.
COELHO, M. Early maturing layers require altered management. Feedstuffs, Minnesota, p. 11-16, June 2001.

FERREIRA, D. F. Sistema de análises de variância para dados balanceados - SISVAR 4.1. Lavras: UFLA, 2000. Pacote computacional.

GERRY, R. W.; BIRD, F. H. The performance of Red x Rock sex links as affected by calcium levels in their growing and laying diets. Poultry Science, Champaign, v. 46, n. 4, p. 1264, Apr. 1967.

LEESON, S.; SUMMERS, J. D. Commercial poultry nutrition. 2. ed. Guelph-Ontario: University Books, 1997.

LOHMANN. Manual de criação e manejo da linhagem Lohmann LSL. Uberlândia: Planalto. 1999.

MEYER, G. B.; BABCOCK, S. W.; SUNDE, M. L. Effect of various prelaying levels of dietary calcium upon subsequent performance in chickens. Poultry Science, Champaign, v. 50, n. 2, p. 536-547, Feb. 1971.

RAO, K. S.; ROLAND, D. A. In vivo limestone solubilization in commercial Leghorns: role of dietary calcium level, limestone particle size, in vitro limestone solubility rate, and the calcium status of the hen. Poultry Science, Champaign, v. 69, n. 12, p. 21702176, Dec. 1990.

ROSTAGNO, H. S.; ALBINO, L. F. T.; DONZELE, J. L.; GOMES, P. C.; FERREIRA, A. S.; OLIVEIRA, R. F.; LOPES, D. C. Tabelas brasileiras para aves e suínos: composição de alimentos e exigências nutricionais. Viçosa: UFV, 2000. 141 p.

SCHOULTEN, N. A. Níveis de cálcio em dietas para frangos de corte suplementadas com fitase. 2001. 79 p. Dissertação (Mestrado em Zootecnia) - Universidade Federal de Lavras, Lavras, 2001.

SIMCO, T. F.; STEPHENSON, E. L. Re-evaluation of the calcium-phosphorus requirents of the chick. Poultry Science, Champaign, v. 40, n. 5, p. 11881192, 1961. 